\title{
Newtonian and general relativistic contribution of gravity to surface tension of strange stars
}

\author{
M. Bagchi ${ }^{1, \star \star \star \star}$, M. Sinha ${ }^{1, \star, \star \star \star}$, M. Dey ${ }^{1, \star, \star \star}$, J. Dey $^{1, \star \star \star}$, and S. Bhowmick ${ }^{2, \star \star}$ \\ ${ }^{1}$ Dept. of Physics, Presidency College, 86/1 College Street, Kolkata 700 073, India \\ e-mail: mnj2003@vsnl.net; kamal1@vsnl.com; deyjm@giascl101.vsnl.net.in \\ 2 Department of Physics, Barasat Govt. College, Barasat, North 24 Parganas, W. Bengal, India
}

Received 1 June 2005 / Accepted 23 July 2005

\begin{abstract}
Surface tension $(S)$ is due to the inward force experienced by particles at the surface and usually gravitation does not play an important role in this force. But in compact stars the gravitational force on the particles is very large and $S$ is found to depend not only on the interactions in the strange quark matter, but also on the structure of the star, i.e. on its mass and radius. Indeed, it has been claimed recently that $511 \mathrm{keV}$ photons observed by the space probe INTEGRAL from the galactic bulge may be due to $\mathrm{e}^{+} \mathrm{e}^{-}$annihilation, and their source may be the positron cloud outside of an antiquark star. Such stars, if they exist, may also go a long way towards explaining away the antibaryon deficit of the universe. For that to happen $S$ must be high enough to allow for survival of quark/antiquark stars born in early stages of the formation of the universe.

High value of $S$ may also assist explanation of delayed $\gamma$-ray burst after a supernova explosion, as conversion from normal matter to strange matter takes place. The possibility of some implications from formation of surface waves are also discussed.
\end{abstract}

Key words. X-rays: binaries - stars: fundamental parameters - relativity - waves - dense matter - equation of state gravitation

\section{Introduction}

For ordinary fluid, $S$ is the property of the specific interactions within and between the media forming an interface, and gravitation does not play any significant role.

Neutron stars models cannot explain the properties of some compact stars. These stars may be made entirely of deconfined $u, d, s$ quark matter (Li et al. 1999a,b) and may have been formed in the early universe in a cosmic separation of phases (Witten 1984). Alternatively they could be produced after supernovae and show observed delayed gamma ray bursts as signals of the transition from a normal matter remnant to a strange one. Both models due to Alcock \& Olinto (1989) and Bombaci et al. (2004, hence forth BPV) need large values of $S$.

The MIT bag and the realistic strange star (ReSS) models are considered. The maximum mass of the star and the corresponding radius obtainable from the MIT bag does not significantly differ from neutron star results. To get objects substantially more compact than neutron stars the ReSS was invoked. The ReSS incorporates chiral symmetry restoration (CSR), in a simple tree level large $N_{\mathrm{c}}$ model, with an interaction which contains both asymptotic freedom (AF) and the confinement

* Visitor (2005), IUCAA, Pune and HRI, Allahabad, India.

$\star \star$ Work supported in part by DST grant No. SP/S2/K-03/2001, Govt. of India.

$\star \star \star$ CSIR NET Fellow, Govt. of India.
- deconfinement mechanism (CDM, Dey et al. 1998). It can explain

1. the properties of compact stars like SAX J1808.8 or 4U 1728-34 (Li et al. 1999a,b);

2. two quasi periodic peaks in X-ray power spectrum of some compact stars (Li et al. 1999; Mukhopadhyay et al. 2003);

3. hours-long superbursts as diquark formation - after pairs are broken due to strong prolonged accretion seen in seven stars (Sinha et al. 2002);

4. breathing mode absorption in 1E 1207-5209 (Sinha et al. 2003) and excess emission bands in six other X-ray emitters (Ray et al. 2004).

The SPI spectrometer on the INTEGRAL satellite has recently detected a bright $511 \mathrm{keV}$ line in the $\gamma$-ray spectrum from the bulge of the galaxy with a spherically symmetric distribution (Knodleseder et al. 2003). This has stimulated research in the fundamental physics that describes cosmological dark matter. Several authors (Boehm et al. 2004; Oaknin \& Zhitnitsky 2004) have suggested that the line comes from positron annihilation, possibly in strange stars formed of antiquarks. The suggestion of cosmic separation of phases (Witten 1984) is that strange stars (SS) can exist from the early universe and can explain dark matter. A crucial condition for the survival of these stars, laid down by Alcock \& Olinto (1989), is a large value of $S$. 
One of the most interesting astrophysical observations of recent times is the occurrence of supernova ( $\mathrm{SN}$ ) with remnants and associated GRB with enormous energy, sometimes immediately following the $\mathrm{SN}$ and sometimes delayed. In a recent paper, BPV considered the possibility that GRBs may be delayed processes following a supernova, in which ordinary matter is converted into strange quark matter (SQM). Importantly, the scenario presented by these authors also suggest that "the existence of compact stars with small radii (quark stars) does not exclude the existence of compact stars with large radii (pure hadronic stars), and vice versa". GRB delay is controlled by "poorly known" $S$ according to these authors. Typical values used in the literature range within $10-50 \mathrm{MeV} \mathrm{fm}^{-2}$ (Heiselberg \& Pethick 1993; Iida \& Sato 1997, 1998). The variation in the transition time from a normal matter remnant to a SQM star could very well be due to a variable $S$, which depends on the size of the SQM star, as we shall argue below.

\section{Surface tension}

We find from simple considerations that $S$ varies from about 10 to $140 \mathrm{MeV} \mathrm{fm}^{-2}$ depending on the star size. The bag model, which was historically the first one used to study SQM gives values for $S$ which is substantially smaller than the ReSS.

In addition to ReSS, in which the interquark potential is due to Richardson, with a QCD scale parameter of $100 \mathrm{MeV}$, we also consider a "new" ReSS (Bagchi et al. 2005) in which two scales are chosen, one for asymptotic freedom $(\sim 100 \mathrm{MeV})$ and the other for confinement $(\sim 350 \mathrm{MeV})$.

The surface at a radius $r$ is $4 \pi r^{2}$, the projection of a quark on it is $\pi r_{n}^{2}-$ if it occupies a sphere of radius $r_{n}=(1 / \pi n)^{1 / 3}$ on the average, where $n$ is the number density. Heiselberg \& Pethick (1993) suggested that the quark scattering cross section $\pi r_{n}^{2}$ can be compared to proton-proton scattering using the quark counting rule $\sigma_{\mathrm{pp}}=3 \sigma_{\mathrm{qq}}=3 \pi r_{n}^{2}$. Following the above relation, we get the value of $\sigma_{\mathrm{pp}}$ to be nearly $25 \mathrm{mb}$ which is quite reasonable as it is quoted to be $25 \mathrm{mb}$ between 140 to $350 \mathrm{MeV}$ (Roy \& Nigam).

This $r_{n}$ is comparable with interparticle separation conventionally defined in nuclear physics for matter, $r_{0}=(3 / 4 \pi n)^{1 / 3}$. $r_{0}$ and $r_{n}$ are compared in Tables 1 and 2.

Considering a SS as a huge drop of SQM, the pressure difference across the surface can be expressed in terms of $S$ :

$|\Delta p|_{r=R}=\frac{2 S}{R}$

At the surface of a SS,

$\Delta p=r_{n} \frac{\mathrm{d} p}{\mathrm{~d} r}$

equivalent to using a cut off $\exp \left(-r / r_{n}\right)$ to obtain the pressure difference at the sharp boundary of the star. One might recall that - unlike a neutron star which needs gravitation to bind it - a SS is self-bound although that does not mean gravitation plays a negligible part in keeping it more compact.

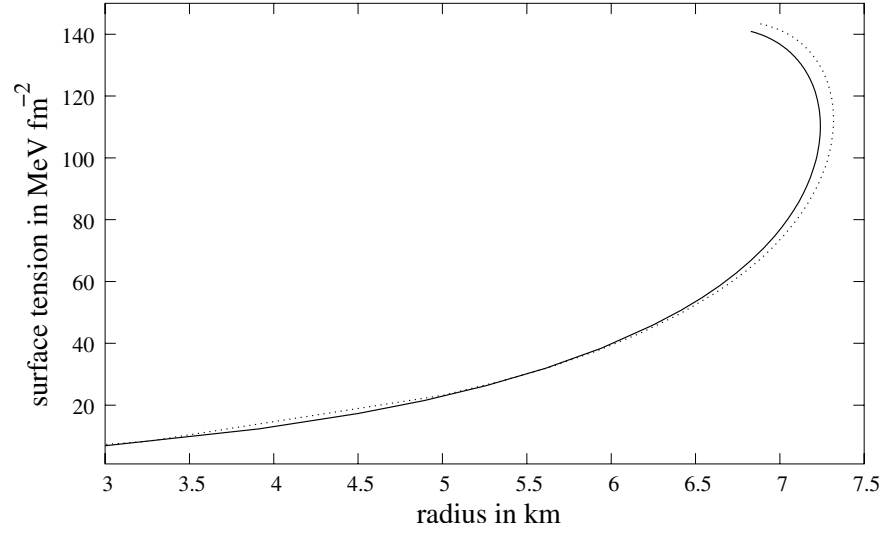

Fig. 1. Variation of $S$ with star size in ReSS and new ReSS. The solid line is for the parameter set ReSS1 and the dotted line for ReSSn1, given in Table 1. $S$ is much larger for both the cases showing that the strength of the interaction is much larger in ReSS compared to the bag model (Fig. 2).

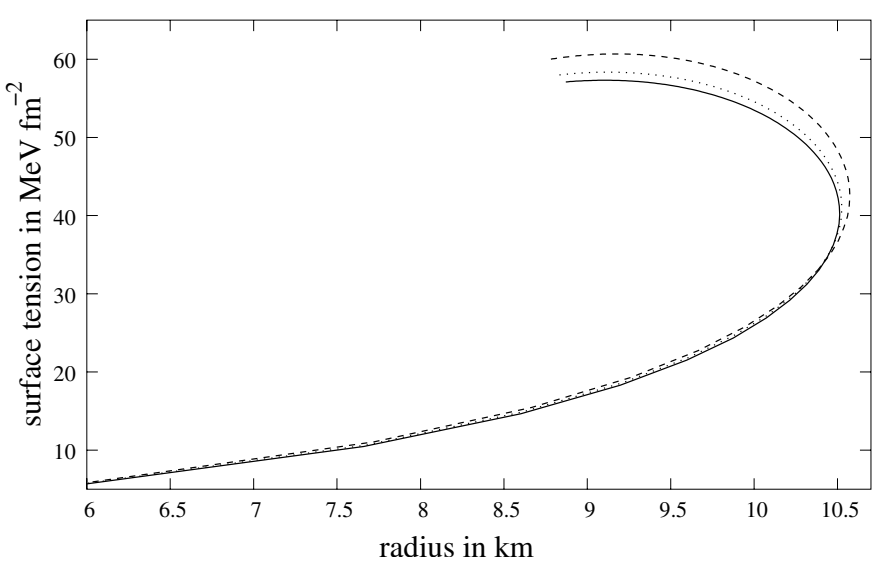

Fig. 2. Variation of $S$ with star size in Bag model for two sets of parameters, showing that $S$ increases appreciably only when $\alpha_{\mathrm{s}}$ is 0.5 . The solid curve is for parameter set bg1, the dotted for bg2 and the dashed for bg3, given in Table 2 .

$\left|\frac{\mathrm{d} p}{\mathrm{~d} r}\right|_{r=R}$ can be obtained by solving the general relativistic equations for hydrostatic equilibrium (TOV equation):

$\frac{\mathrm{d} p}{\mathrm{~d} r}=\frac{-G[p(r)+\epsilon]\left[m(r)+4 \pi r^{3} p(r)\right]}{r^{2}\left[1-\frac{2 G m(r)}{r}\right]}$.

One gets $S \sim 140 \mathrm{MeV} \mathrm{fm}^{-2}$ for a SQM star of radius $6.95 \mathrm{~km}$ and mass of $1.42 M_{\odot}$ (see Fig. 1). The Newtonian limit (Chiu 1968) leads to $S \sim 36 \mathrm{MeV} \mathrm{fm}^{-2}$ for a star of same radius. This shows that the general relativistic effect is very important. It can also be seen from Fig. 1 for radii 3 to $6 \mathrm{~km}$ one gets the standard value of $S$ varying from 10 to $50 \mathrm{MeV} \mathrm{fm}^{-2}$, showing that our estimate of $r_{n}$ is realistic. The bag model gives more moderate values for $S$ which is understood as being due to the less compact nature of the star (see Fig. 2). For both tables the strange quark has an additional mass of $150 \mathrm{MeV}$.

In the graphs, we have chosen $\mathrm{MeV} \mathrm{fm}{ }^{-2}$ as the unit of $S$; while in the tables $\mathrm{MeV}^{3}$ is the chosen unit for surface tension as given by Alcock \& Olinto (1989) to facilitate comparison with them. They already indicated that lumps of SQM might have survived dissolution into hadrons in the early universe if $S$ 
Table 1. Results from ReSS and new ReSS models. The central density is $\rho_{\mathrm{c}}$ and $\alpha_{\mathrm{s}}$ is the strong coupling constant that controls the Debye screening. The parameter $N$ controls the CSR, through the quark mass equation, $m=m_{\mathrm{q}} \operatorname{sech}\left(\frac{1}{N} n_{\mathrm{B}}\right)$, where $n_{\mathrm{B}}=\left(n_{\mathrm{u}}+n_{\mathrm{d}}+n_{\mathrm{s}}\right) / 3$ is the baryon number density and $n_{0}$ is the normal nuclear matter density. The strange quark mass $m_{\mathrm{s}}=m+150 \mathrm{MeV}$.

\begin{tabular}{ccccccccccc}
\hline \hline & \multicolumn{1}{c}{ Parameters } & \multicolumn{7}{c}{ Maximum mass and corresponding results } \\
\hline EOS & $N$ & $\begin{array}{c}m_{\mathrm{q}} \\
\mathrm{MeV}\end{array}$ & $\alpha_{\mathrm{s}}$ & $\begin{array}{c}\text { Mass } \\
M_{\odot}\end{array}$ & $\begin{array}{c}\rho_{\mathrm{c}} \\
10^{14} \mathrm{gm} / \mathrm{cm}^{3}\end{array}$ & $\begin{array}{c}\text { Radius } \\
\mathrm{km}\end{array}$ & $\begin{array}{c}\left|\frac{\mathrm{d} p}{\mathrm{~d} r}\right|_{r=R} \\
\mathrm{MeV} / \mathrm{fm}^{3} / \mathrm{km}\end{array}$ & $\begin{array}{c}r_{n} \\
\mathrm{fm}\end{array}$ & $\begin{array}{c}r_{0} \\
\mathrm{fm}\end{array}$ & $\begin{array}{c}S \\
\mathrm{MeV}^{3}\end{array}$ \\
\hline ReSS & 3.0 & 310 & 0.2 & 1.44 & 46.85 & 7.06 & 73.87 & 0.51 & 0.47 & $(173.5)^{3}$ \\
$\operatorname{ReSSn}$ & 3.0 & 325 & 0.65 & 1.47 & 45.49 & 7.14 & 73.05 & 0.52 & 0.47 & $(173.9)^{3}$ \\
\hline
\end{tabular}

Table 2. Parameters and results for bag models. The masses $m_{\mathrm{u}}=0=m_{\mathrm{d}}$ and $m_{\mathrm{s}}=150 \mathrm{MeV}$.

\begin{tabular}{cccccccccc}
\hline \hline & \multicolumn{2}{c}{ Parameters } & \multicolumn{7}{c}{ Maximum mass and corresponding results } \\
\hline EOS & $\begin{array}{c}B \\
\mathrm{MeV} / \mathrm{fm}^{3}\end{array}$ & $\alpha_{\mathrm{s}}$ & $\begin{array}{c}\text { Mass } \\
M_{\odot}\end{array}$ & $\begin{array}{c}\rho_{\mathrm{c}} \\
10^{14} \mathrm{gm} / \mathrm{cm}^{3}\end{array}$ & $\begin{array}{c}\text { Radius } \\
\mathrm{km}\end{array}$ & $\begin{array}{c}\left|\frac{\mathrm{d} p}{\mathrm{~d} r}\right|_{r=R} \\
\mathrm{MeV} / \mathrm{fm}^{3} / \mathrm{km}\end{array}$ & $\begin{array}{c}r_{n} \\
\mathrm{fm}\end{array}$ & $\begin{array}{c}r_{0} \\
\mathrm{fm}\end{array}$ & $\begin{array}{c}S \\
\mathrm{MeV}^{3}\end{array}$ \\
\hline $\mathrm{bg} 1$ & 60 & 0.0 & 1.81 & 23.17 & 10.07 & 14.75 & 0.71 & 0.64 & $(123.1)^{3}$ \\
$\mathrm{bg} 2$ & 60 & 0.17 & 1.82 & 23.18 & 10.08 & 14.93 & 0.71 & 0.65 & $(123.1)^{3}$ \\
bg3 & 60 & 0.50 & 1.85 & 23.20 & 10.12 & 15.32 & 0.72 & 0.66 & $(125.6)^{3}$ \\
bg4 & 60 & 0.17 & 1.75 & 24.85 & 9.76 & 15.61 & 0.71 & 0.65 & $(126.1)^{3}$ \\
bg5 & 75 & 0.17 & 1.64 & 28.81 & 9.06 & 20.71 & 0.68 & 0.61 & $(130.9)^{3}$ \\
\hline
\end{tabular}

is around $(178 \mathrm{MeV})^{3}$. The $S$ calculated from ReSS and new ReSS gives results of this order while that from the bag model is low.

\section{Other implications}

Type 1 X-ray bursts from seven LMXBs have been well studied through RXTE. Often a peak is observed in the power density spectrum (PDS) at a frequency which is taken as the compact star's spin frequency (or half of the spin frequency). But astonishingly, the frequency is not constant throughout the burst. It shifts towards a higher value. There are several possible explanation such as photospheric radius expansion (Strohmayer et al. 1997), Rossby mode vibration (Heyl 2004) etc. We try to explain it as the onset of a surface wave as the burst proceeds and the shift of the peak is a result of the coupling of the surface wave to the burst power.

Applying Bernoulli's theorem to the crest and trough of the sinusoidal wave, we get the equation:

$$
\begin{aligned}
& \frac{G M(R+h)}{(R+h)^{2}-R_{\mathrm{s}}{ }^{2}}+\frac{1}{2}\left(V+\frac{2 \pi b}{T}\right)^{2}-\frac{S c^{2}}{b \epsilon}= \\
& \frac{G M(R-h)}{(R-h)^{2}-R_{\mathrm{s}}{ }^{2}}+\frac{1}{2}\left(V-\frac{2 \pi b}{T}\right)^{2}+\frac{S c^{2}}{b \epsilon}
\end{aligned}
$$

where $S, M, R, R_{\mathrm{s}}, c$ are the surface tension, mass, radius, the Schwarzchild radius of the star and velocity of light. $\epsilon$ is the energy density at the star surface. $h, T, V$ are respectively the amplitude, time period and velocity of the wave. The radius of curvature at crest and trough, $b$, is given by:

$b=\frac{\left(1+(\mathrm{d} y / \mathrm{d} x)^{2}\right)^{3 / 2}}{\mathrm{~d}^{2} y / \mathrm{d} x^{2}}=\frac{\lambda^{2}}{4 \pi^{2} h}=\frac{V^{2} T^{2}}{4 \pi^{2} h}$.
With the correction term $2 \pi b / T$ in the velocity, $V$, due to circular motion of the particle, the Eq. (4) reduces to:

$$
\begin{aligned}
\frac{\pi h V^{2} R_{\mathrm{s}} c^{2}}{2 T}\left[\frac{(R+h)}{(R+h)^{2}-{R_{\mathrm{s}}}^{2}}\right. & \left.-\frac{(R-h)}{(R-h)^{2}-{R_{\mathrm{s}}}^{2}}\right]= \\
- & {\left[V^{5}+\frac{8 \pi^{3} h^{2} S c^{2}}{\epsilon T^{3}}\right] . }
\end{aligned}
$$

We solve Eq. (6) graphically; both R.H.S and L.H.S. are functions of $V, h$ and $T$ - they can be denoted by $A(V, h, T)$ and $B(V, h, T)$. Different values of $V$ can be obtained for combinations of $h$ and $T$. Figure 3 shows one such solution with $T=2 / 3 \mathrm{~s}, V=36.89 \mathrm{~km} \mathrm{~s}^{-1}$ giving $h=64.76 \mathrm{~cm}$.

To fix $V$ and $T$ we turn to the burst from the compact star 4U 1728-34 (Strohmayer et al. 1996; Strohmayer et al. 1998), the rise time of the burst is $0.6 \mathrm{~s}$ and the frequency shift $(\Delta f)$ observed in the maximum of PDS is $1.5 \mathrm{~Hz}$. Assuming the surface wave originates at the burst spot and travels over the entire surface of the star within the burst rise time - its velocity is $36.89 \mathrm{~km} \mathrm{~s}^{-1}$ for a star with half the circumference of $7.05 \pi \mathrm{km}$. We take the time period of the wave as the inverse of the frequency shift i.e., $T=2 / 3$ s. From these values of $V$ and $T$, we get $h=64.75 \mathrm{~cm}$ from Fig. 3. These values are therefore consistent. For theoretical interest, in Fig. 4 we plot the velocity of the wave for a wide range of the amplitude, keeping $T$ fixed at 2/3 s. In Fig. 5, we plot the variation of $V$ with $h$ for different values of $T$, keeping $h$ in order of $\mathrm{cm}$ and $T$ also near to $2 / 3 \mathrm{~s}$.

We thank the anonymous referee for reminding us that a SS has an electron cloud outside it and this leads to a electrostatic field which may be as strong as $10^{17} \mathrm{~V} \mathrm{~cm}^{-1}$ decreasing to $10^{11}$ as one goes out radially by one $\AA$ (see Xu et al. 2001, for a review and update). This may affect the above estimate and will be considered elsewhere. The accretion rate on stars 


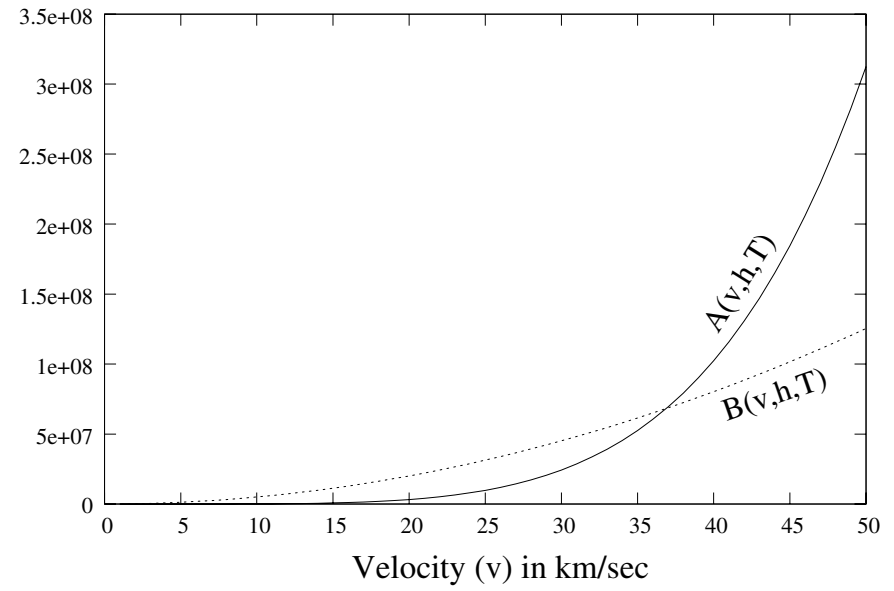

Fig. 3. Solution of Eq. (6) for $V=36.89 \mathrm{~km} \mathrm{~s}^{-1}$ and $T=2 / 3 \mathrm{~s}$.

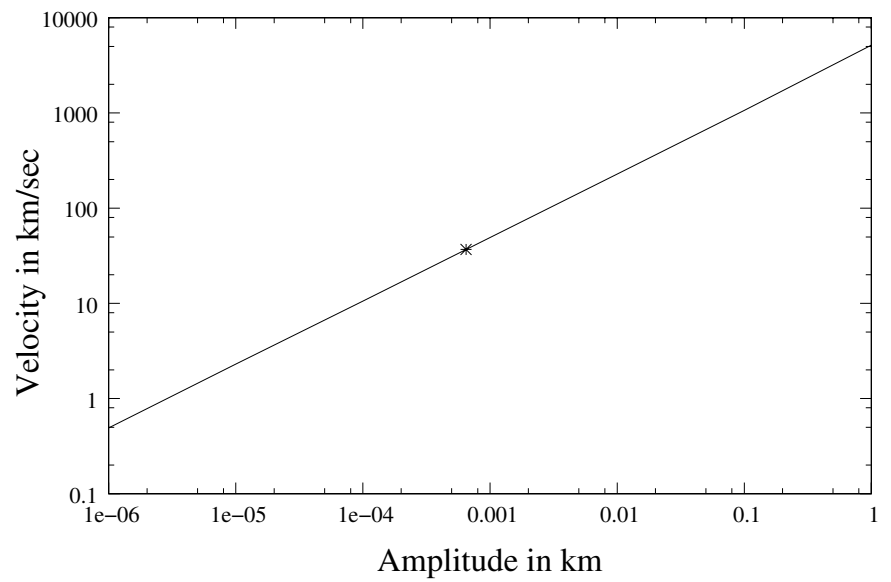

Fig. 4. Variation of $V$ with $h$ for a wide range of values of $h$, keeping $T$ fixed at $2 / 3 \mathrm{~s}$. The $*$ point denotes the set of $V, h$ required to explain the $\Delta f$, observed in $4 \mathrm{U} 1728-34$.

vary a lot, the maximum being $10^{-5} M_{\odot} / \mathrm{yr}$ and this has to be taken into account. Finally, the surface wave may cause gravitational radiation which should be observable in the future. The amplitude of the surface wave that we have estimated is of order $50 \sim 100 \mathrm{~cm}$, as such it will not cause serious deformation of the shape of a $R=7 \mathrm{~km}$ star.

\section{Conclusions and summary}

The high values of $S$ found for the ReSS and new ReSS keep open the possibility of cosmic separation in the early universe, which in turn may explain the baryon-antibaryon asymmetry and the dark matter problem according to Oaknin \& Zhitnitsky (2004). The large value of surface tension could also help in explaining the variable delay time between a SN and a GRB in the scenario of BPV. It is amusing to find that $S$ in dense systems do after all depend on gravitation. With our estimated value of $S$, we can also explain the frequency shift in the PDS of type $1 \mathrm{X}$-ray burst.

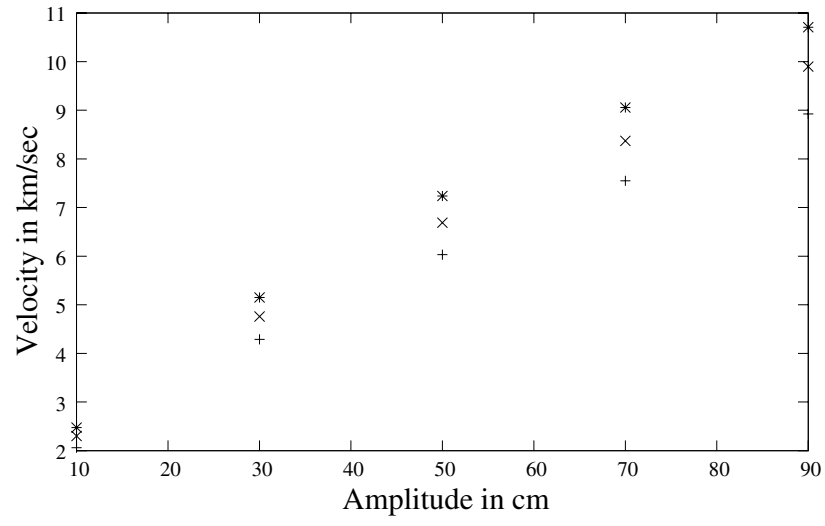

Fig. 5. Variation of $V$ with $h$ for different $T$. The $*$ 's are for $T=$ $0.52632 \mathrm{~s}(\Delta f=1.9)$, the $\times$ 's are for $T=2 / 3 \mathrm{~s}(\Delta f=1.5)$ and the +'s are for $T=0.90909 \mathrm{~s}(\Delta f=1.1)$.

Acknowledgements. M.B. checked the results of $4 \mathrm{U} 1728-34$ at TIFR thanks to the hospitality of Dr. Biswajit Paul and thanks Uddipan Mukherjee for help.

\section{References}

Alcock, C., \& Olinto, A. 1989, Phys. Rev. D, 39, 1233

Bagchi, M., Ray, S., Dey, M., \& Dey, J. 2005, A\&A, submitted

Boehm, C., Hooper, D., Silk, J., Casse, M., \& Paul, J. 2004, Phys. Rev. Lett., 92, 101301

Bombaci, I., Parenti, I., \& Vidaña, I. 2004, ApJ, 614, 314

Chiu, H. Y. 1968, Stellar Physics (Blaisdell Publishing Company), 70

Dey, M., Bombaci, I., Dey, J., Ray, S., \& Samanta, B. C. 1998, Phys. Lett. B, 438, 123; Addendum 1999, 447, 352; Erratum B 1999 467, 303; Indian J. Phys. B 1999, 73, 377

Heiselberg, H., \& Pethick, C. J. 1993, Phys. Rev. D, 48, 2916

Heyl, J. S. 2004, ApJ, 600, 939

Iida, K., \& Sato, K. 1997, Prog. Theo. Phys., 98, 277

Iida, K., \& Sato, K. 1998, Phys. Rev. C, 58, 2538

Knodleseder, J., Lonjou, V., Jean, P., et al. 2003, A\&A, 2003, 411, L457

Li, X., Bombaci, I., Dey, M., Dey, J., \& van den Heuvel, E. P. J. 1999a, Phys. Rev. Lett., 83, 3776

Li, X., Ray, S., Dey, J., Dey, M., \& Bombaci, I. 1999b, ApJ, 527, L51

Mukhopadhyay, B., Ray, S., Dey, J., \& Dey, M. 2003, ApJ, 584, L83

Oaknin, D. H., \& Zhitnitsky, A. R. 2005, Phys. Rev. Lett., 94, 101301

Ray, S., Dey, J., Dey, M., \& Bhowmick, S. 2004, MNRAS, 353, 825

Roy, R. R., \& Nigam, B. P. 1996, Nuclear Physics: Theory and Experiment (New Delhi: New Age International Publishers), 89

Sinha, M., Dey, M., Ray, S., \& Dey, J. 2002, MNRAS, 337, 1368

Sinha, M., Dey, J., Dey, M., Ray, S., \& Bhowmick, S. 2003, Mod. Phys. Lett. A, 18, 661

Strohmayer, T. E., Zhang, W., Swank, J. H., Titarchuk, L., \& Day, C. 1996, ApJ, 469, L9

Strohmayer, T. E., Jahoda, K., Giles, A. B., \& Lee, U. 1997, ApJ, 486, 355

Strohmayer, T. E., Zhang, W., Swank, J. H., \& White, N. E. 1998, ApJ, 498, L135

Witten, E. 1984, Phys. Rev. D, 30, 272

Xu, R. X., Zhang, B., \& Qiao, G. J. 2001, Astropart. Phys., 15, 101 